ionization-time-of-flight mass spectrometry was used to identify the specimen, resulting in a score of 1.77 for L. felleii. No reaction during the serological aggregation test (Denka Seiken, Tokyo) was observed against L. pneumophila serogroup (SG) 1-SG6, L. bozemanii, L. dumoffii, Legionella gormanii, or L. micdadei.

This study is the first report to describe the Legionella contamination of water taps in a brand-new building, before the start of standard usage. Water taps, showers, sinks, and water systems in healthcare facilities have been recognized to be the causes of healthcare-associated legionellosis. ${ }^{3-5}$ Previous studies have not considered the possibility that even brand-new hospital water systems may be at risk for Legionella spp colonization during the building process, which can persist and spread once the hospital is operational. Although Legionella spp contamination in water systems has been associated with water scale, stagnant water, and sediment, brand-new buildings, even prior to active use, may possess contamination risk factors, such as stagnant dead spaces. ${ }^{3}$ This study indicates that we must pay attention to the risks of healthcare-associated waterborne infections, even in brand-new buildings and before patients move in.
Acknowledgements. We thank Lisa Giles, PhD, from Cambridge Proofreading (https://proofreading.org/) for editing a draft of this manuscript.

Financial support. No financial support was provided relevant to this article.

Conflicts of interest. The author declares no conflicts of interest.

\section{References}

1. Soda EA, Barskey AE, Shah PP, et al. Vital signs: healthcare-associated Legionnaires' disease surveillance data from 20 states and a large metropolitan area-United States, 2015. Am J Transplant 2017;17:2215-2220.

2. Amemura-Maekawa J, Kura F, Chida K, et al. Legionella pneumophila and other Legionella species isolated from legionellosis patients in Japan between 2008 and 2016. Appl Environ Microbiol 2018;84:e00721-18.

3. Decker BK, Palmore TN. The role of water in healthcare-associated infections. Curr Opin Infect Dis 2013;26:345-351.

4. Kanamori H, Weber DJ, Rutala WA. Healthcare outbreaks associated with a water reservoir and infection prevention strategies. Clin Infect Dis 2016;62:1423-1435.

5. Laganà $\mathrm{P}$, Caruso G, Piccione $\mathrm{D}$, et al. Legionella spp, amoebae and notfermenting gram-negative bacteria in an Italian university hospital water system. Ann Agric Environ Med 2014;21:489-493.

\title{
Reply to "Comparative evaluation of the microbicidal activity of low-temperature sterilization technologies to steam sterilization"
}

Randal Eveland PhD, MS (1)

Steris, Mentor, Ohio

To the Editor-This letter is in response to the article by Rutala et $\mathrm{al}^{1}$ that compared the microbial kill of steam, ethylene oxide (ETO), hydrogen peroxide gas plasma (HPGP), and vaporized hydrogen peroxide (VHP) in the presence of salt and serum in standard sterilization cycles.

Unfortunately, at this time, there are no 'standard' gaseous hydrogen peroxide sterilization processes. The article fails to consider that although both HPGP and VHP processes use gaseous hydrogen peroxide as the sterilant, the processes are distinct and different in the way they operate. Even though 28-minute HPGP and VHP cycles are used, these cycles use significantly different concentrations of sterilant. The HPGP exposure is $25.6 \mathrm{mg} / \mathrm{L}$ $\mathrm{H}_{2} \mathrm{O}_{2}$ for 7 minutes whereas the VHP exposure is $9.1 \mathrm{mg} / \mathrm{L} \mathrm{H}_{2} \mathrm{O}_{2}$ for 12 minutes. The importance of disinfectant concentration is explained in the 2008 CDC Guideline for Disinfection and Sterilization in Healthcare Facilities where it is stated that "The more concentrated the disinfectant, the greater is its efficacy and the shorter the time necessary to achieve microbial kill." ${ }^{2}$ For these evaluations with no chamber load, sterilant concentration should have been considered.

The delineation of the gaseous hydrogen peroxide processes like HPGP and VHP, with the subsequent comparisons of efficacy

Author for correspondence: Randal Eveland, E-mail: randal_eveland@steris.com

Cite this article: Eveland R. (2020). Reply to "Comparative evaluation of the microbicidal activity of low-temperature sterilization technologies to steam sterilization". Infection Control \& Hospital Epidemiology, 41: 999-1000, https://doi.org/10.1017/ ice. 2020.122 minus any consideration of sterilant concentration, seems to imply that there is a benefit from plasma within the sterilization process. This contention contradicts the current understanding of the purpose of a gas plasma in HPGP systems, in which it is known that the plasma step has little to no contribution to sterilizer efficacy. In the only research ever published to evaluate the impact of plasma in a HPGP process, the plasma phase appeared to be nonsporicidal. ${ }^{3}$

The detoxifying (residual sterilant removing) effect of the plasma would have no impact on gaseous hydrogen peroxide microbial lethality; thus, the $\sim 3$-fold sterilant concentration difference (25.6 vs $9.1 \mathrm{mg} / \mathrm{L} \mathrm{H}_{2} \mathrm{O}_{2}$ for the HPGP and VHP systems, respectively) is clearly responsible for the observed efficacy differences in HPGP and VHP processes. Higher concentration is not always beneficial. Beyond efficacy, hospitals also consider the gentleness of the sterilization process to include the potential impact of higher sterilant concentrations and higher sterilant dose on device material compatibility (especially devices susceptible to reaction with the highly oxidizing hydrogen peroxide sterilant) or device biocompatibility as well as the potential impact of plasma on medical device surfaces.

Both the HPGP and VHP sterilization cycles have been cleared by the Food and Drug Administration (FDA), so both have demonstrated the ability to achieve a sterility assurance level (SAL) of 10E-6 for their claimed processes. The CDC disinfection guidelines ${ }^{2}$ specify that even salts dissolved within surrogate body fluids dissolve with 60 seconds of nonflowing water; therefore, showing 
that, from a use perspective, the protective nature of salt has little clinical relevance. Although salt has been shown historically by many investigators to potentially impede hospital sterilization of medical devices, the emphasis of these results should be to highlight the need for thorough cleaning methodologies.

Acknowledgments. None.

Financial support. No financial support was provided relevant to this article.

Conflicts of interest. All authors report no conflicts of interest relevant to this article.

\section{References}

1. Rutala WA, Gergen MF, Sickbert-Bennett EE, Weber DJ. Comparative evaluation of the microbicidal activity of low-temperature sterilization technologies to steam sterilization. Infect Control Hosp Epidemiol 2020 [Epub ahead of print]. doi: 10.1017/ice.2020.2.

2. Guideline for disinfection and sterilization in healthcare facilities, 2008, updated May 2019. Centers for Disease Control and Prevention website. https://www.cdc.gov/infectioncontrol/pdf/guidelines/disinfection-guidelinesH.pdf. Published 2019. Accessed April 13, 2020.

3. Krebs MC, Becasse P, Verjat D, Darbord JC, Gas-plasma sterilization: relative efficacy of the hydrogen peroxide phase compared with that of the plasma phase. Int J Pharm 1998;160:75-81.

\title{
Reply to Randal W. Eveland regarding comparative evaluation of the microbicidal activity of low-temperature sterilization technologies to steam sterilization
}

\author{
William A. Rutala PhD, MPH${ }^{1}$, Maria F. Gergen MT(ASCP) ${ }^{3}$, Emily E. Sickbert-Bennett PhD, $\mathrm{MS}^{1,2}$ and \\ David J. Weber MD, MPH ${ }^{1,2}$ \\ ${ }^{1}$ Division of Infectious Diseases, University of North Carolina School of Medicine, Chapel Hill, North Carolina, ${ }^{2}$ Department of Hospital Epidemiology, University of \\ North Carolina Health Care, Chapel Hill, North Carolina and ${ }^{3}$ Formerly Department of Hospital Epidemiology, University of North Carolina Health Care, Chapel \\ Hill, North Carolina
}

To the Editor-We thank Dr Randal Eveland, Steris Corporation, for his letter regarding our paper that compared the microbicidal activity of low-temperature sterilization technologies (ie, vaporized hydrogen peroxide [VHP], ethylene oxide [ETO], and hydrogen peroxide gas plasma [HPGP]) to steam sterilization in the presence of salt and serum to simulate inadequate precleaning. ${ }^{1}$ As noted in our paper, the literature contains a paucity of information on the comparative microbicidal activity of the sterilization technologies cleared by the Food and Drug Administration (FDA) for sterilizing medical and surgical devices. We believe that the data from this study will help clinicians in infection prevention assess the robustness of healthcare sterilization technologies and the risk of infection to patients when an uncleaned instrument is unintentionally brought into the operating room or used on a patient.

We agree with Dr Eveland there are differences in concentration and duration of the VHP and HPGP cycles. Our experiments compared the microbicidal activity of FDA-cleared, low-temperature sterilization technologies to steam sterilization in the presence of salt and serum. The addition of salt and serum simulated inadequate cleaning of instruments prior to sterilization. We evaluated the "robustness" of sterilization technology that is used by hospitals throughout the United States. Robustness is defined as the ability to withstand and overcome adverse conditions or rigorous testing.

Concerning plasma in the HPGP technology, our intention was not to define the components of the cycle that created the robustness (eg, higher concentrations of hydrogen peroxide, plasma); it was

Author for correspondence: William A. Rutala, E-mail: brutala@med.unc.edu Cite this article: Rutala WA, et al. (2020). Reply to Randal W. Eveland regarding comparative evaluation of the microbicidal activity of low-temperature sterilization technologies to steam sterilization. Infection Control \& Hospital Epidemiology, 41: 1000-1001, https://doi.org/10.1017/ice.2020.239 solely to define whether FDA-cleared sterilization technologies had the same robustness or ability to inactivate microorganisms in the presence of organic matter and salt. Our results demonstrated that some sterilization technologies were more "forgiving" or safe when cleaning is not complete. Because protein (organic matter) remains on cleaned surgical instruments, ${ }^{2}$ we must investigate at what point the presence of protein overwhelms the ability of the sterilizer to inactivate contaminating microorganisms. Alternatively, we should consider using the most robust sterilization technologies that inactivate microorganisms in the presence of organic matter and salt when possible.

Regarding the comparison of HPGP to VHP and materials compatibility, there are other factors involved in materials compatibility than the hydrogen peroxide concentration alone. Although the theoretical concentration of hydrogen peroxide for HPGP is higher than for VHP (ie, 25.6 vs $9.1 \mathrm{mg} / \mathrm{L}$ hydrogen peroxide for the HPGP and VHP, respectively), the plasma process quickly removes the hydrogen peroxide from the load by dissociating unreacted hydrogen peroxide into oxygen and water, eliminating the need for aeration. ${ }^{3}$ The VHP sterilizer passes hydrogen peroxide through a catalytic converter where it is reduced to water and oxygen. The HPGP system has 3 potential advantages. First, because the plasma quickly removes the residual hydrogen peroxide, rather than a gradual release with VHP, there may be improved material compatibility and biocompatibility. However, we have not been able to find any data on the internet or in the peer-reviewed literature that demonstrated that VHP is more or less materials compatible or biocompatible than HPGP. Second, regarding environmental hydrogen peroxide levels, for both sterilizers there were no notable emissions from the sterilizers during the cycle. However, other investigators measured significant hydrogen peroxide emissions when the VHP chamber door was open compared 